\title{
CONSULTORÍA DE ENFERMERÍA EN SALUD MENTAL PARA ANTENCIÓN PRIMARIA
}

\author{
Ana GARCÍA LABORDA \\ Enfermera Psiquiátrica. Socióloga \\ Centro de Salud Mental de Parla \\ Dirección de contacto: C/ Pablo Sorozábal 4 \\ 28980 PARLA (MADRID) \\ Email: anag28@smail1.ocenf.org
}

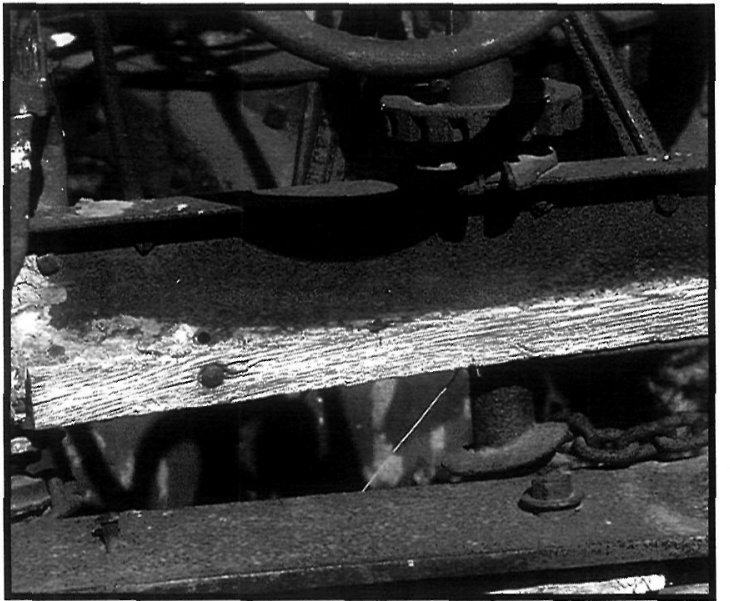

MENTAL HEALTH NURSING CONSULTANCY FOR PRIMARY HEALTH CARE.

$\Gamma$ his article describes a research-action experience whose aim was implementing a support programme for the diagnose of selfconcept. The purpose is to state the viability of the consultant nurse as a figure within the health care system.

The used methodology integrates a project in three phases: project of quality improvement in order to find out the reasons that prevent the diagnose of self-concept from being implemented, intervention on these reasons, and a new project of improvement for the final evaluation of the diagnose implementation.

Key words: Quality improvement. Nursing diagnose. Orem model. Self-concept. Support programme.

\section{RESUMEN}

71 artículo expone una experiencia de investigación-acción con objeto de instaurar un programa de apoyo al diagnóstico de autoconcepto. Su objetivo es constatar la viabilidad de la figura de la enfermera consultora en el sistema sanitario.

La metodología utilizada integra un proyecto en tres fases: proyecto de mejora de calidad con objetivo de dilucidar las causas que dificultan la implantación del diagnóstico de Autoconcepto, intervención sobre las causas y un nuevo proyecto de mejora para la evaluación final de la implantación del diagnostico.

Palabras clave: Mejora de calidad. Diagnóstico de Enfermería. Modelo Orem. Autoconcepto. Programa de Apoyo.

El presente trabajo se diseñó como un proyecto de mejora de calidad, tratando de aunar dos tendencias que han demostrado su utilidad para abordar de manera eficiente desde el sistema sanitario los procesos de salud-enfermedad. El primer aspecto es elegir como sistema de abordaje un proyecto de mejora inscrito la filosofía de Gestión de Calidad Total para racionalizar todos y cada uno de los pasos del proceso de actuación como profesionales sanitarios. El segundo aspecto, aunque igualmente relevante, es utilizar como instrumento de comprensión y abordaje de la actividad de cuidar, un modelo genuinamente enfermero, que permite dar cuenta de todo el proceso de cuidar.

Además el trabajo hace una propuesta poco desarrollada en nuestro país, y es la posibilidad y utilidad de disponer de enfermeras consultoras, 
que con su apoyo y asesoría permitan a las enfermeras generalistas aumentar su repertorio de habilidades en la agencia de autocuidados.

\section{ANTECEDENTES}

En febrero de 1998, toda la enfermería de Atención Primaria del Área 10 del Insalud de Madrid empieza a trabajar con metodología diagnóstica según el modelo Orem. Dadas las dificultades observadas empíricamente, en el área diagnóstica de autoconcepto, se elaboró un Proyecto de mejora de calidad, que es el que voy a exponer a continuación.

\section{PROYECTO DE MEJORA DE CALIDAD}

\section{1. - Título del estudio:}

Evaluación del diagnóstico de autoconcepto en enfermería.

\section{2. - Definición del problema y oportunidad} de mejora:

Hay un bajo número de diagnóstico de enfermería en el Área de Autoconcepto en los centros de salud de Parla: en el periodo de 1-2-98 a 30-9-98 se realizaron un total de 70 , un $6,1 \%$ respecto al resto de diagnósticos de enfermería. Esto supone un cierto descuido de los aspectos psicológicos en la actividad de enfermería en Atención Primaria.

\section{3. - Equipo de evaluación y mejora de la calidad}

\begin{tabular}{|l|l|}
\hline \multicolumn{1}{|c|}{ Miembros } & \multicolumn{1}{c|}{ Funciones } \\
\hline$-\quad$ Enfermeras E.A.P. "Isabel II - 1" & \\
- Enfermeras E.A.P. "Isabel II - 2" & $-\quad$ Realización y registro de datos. \\
\hline Enfermeras E.A.P. "Pintores" & $\begin{array}{l}\text { Diseñar el proyecto de calidad. } \\
\text { Realizar la } 1^{\text {a }} \text { evaluación y la } \\
\text { reevaluación } \\
\text { Poner en marcha las medidas } \\
\text { correctoras. } \\
\text { Mental de Parla. }\end{array}$ \\
\hline
\end{tabular}

\section{4. - Cronograma}

\begin{tabular}{|c|c|c|c|c|c|c|c|c|c|c|c|c|c|}
\hline Fecha de inicio 1-9-98 & Fecha conclusión 30-5-99 & \multicolumn{12}{|c|}{ SEMANAS } \\
\hline TAREA & RESPONSABLE & 1 & 2 & 3 & 4 & 5 & 6 & 7 & 8 & 9 & 10 & 11 & 12 \\
\hline Identificación oportunidad de mejora & Enfermera de Salud Mental & & & & & & & & & & & & \\
\hline Objetivos del estudio & Enfermera de Salud Mental & & & & & & & & & & & & \\
\hline Análisis de la oportunidad de mejora & Enfermera de Salud Mental & & & & & & & & & & & & \\
\hline Diseño del estudio de evaluación & Enfermera de Salud Mental & & & & & & & & & & & & \\
\hline Análisis y presentación de datos eval. & Enfermera de Salud Mental & & & & & & & & & & & & \\
\hline \multirow{3}{*}{ Diseño de la intervención } & Enfermera de Salud Mental & & & & & & & & & & & & \\
\hline & & \multicolumn{12}{|c|}{ MESES } \\
\hline & & 1 & 2 & 3 & 4 & 5 & 6 & 7 & 8 & 9 & 10 & 11 & laño \\
\hline Reevaluación & Enfermera de Salud Mental & & & & & & & & & & & & \\
\hline
\end{tabular}




\section{5. - Objetivos del estudio del nivel de calidad}

Incrementar el número total de diagnósticos de enfermería en el área de autoconcepto un $10 \%$ el periodo comprendido entre octubre de 1998 y mayo de 1999.

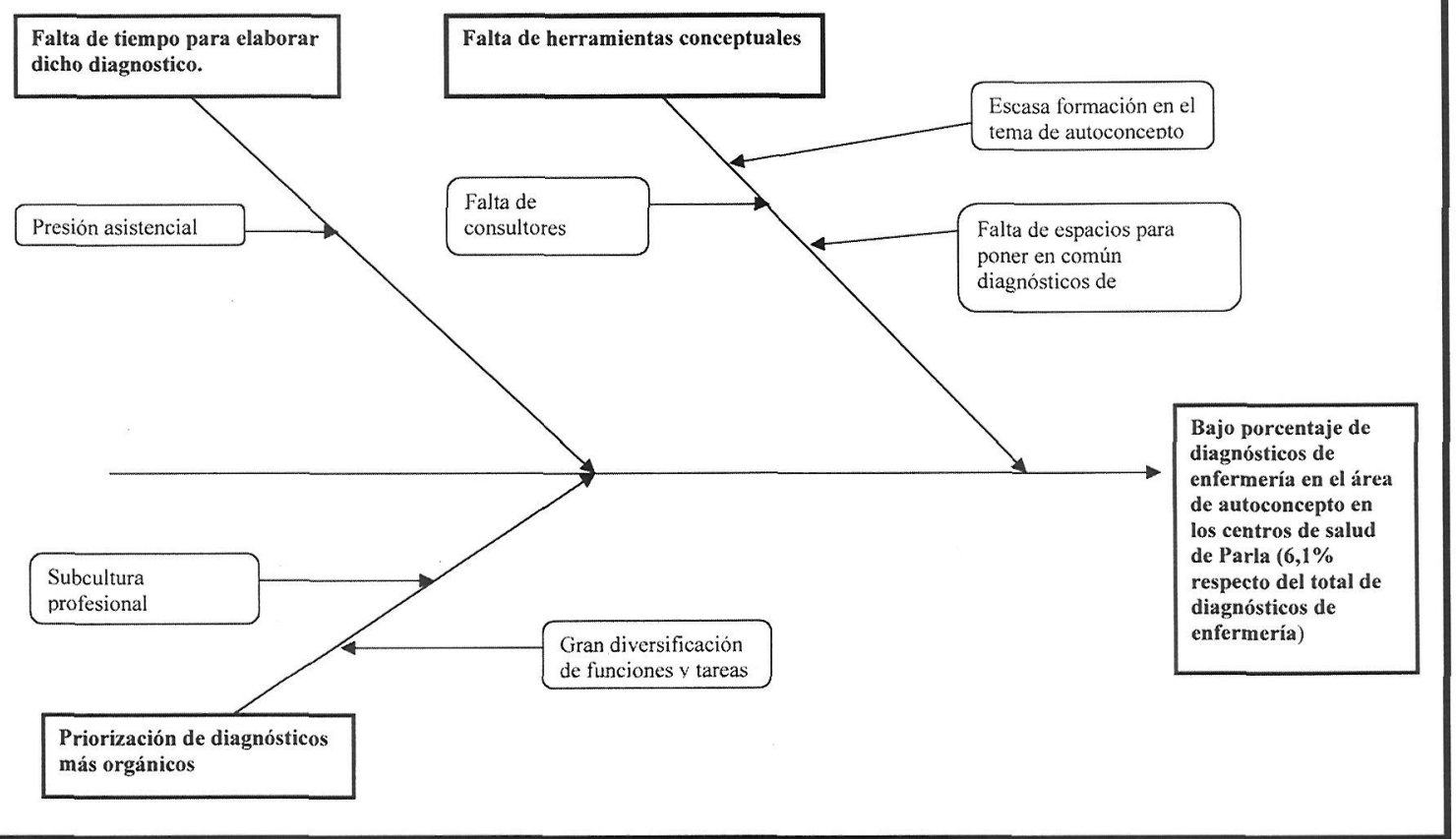

\section{6. - Análisis de causas:}

Diagrama de espina de pescado.

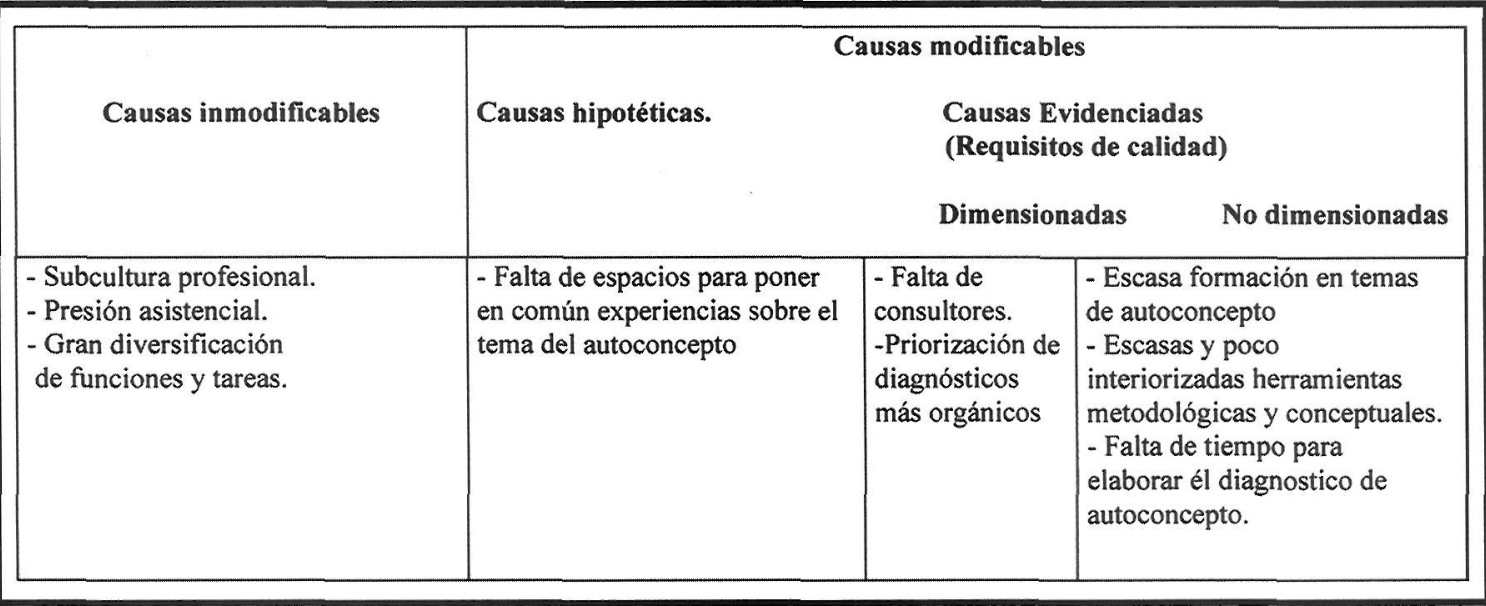




\begin{tabular}{|l|l|}
\hline \multicolumn{1}{|c|}{ Causas sobre las que es posible actuar } & \multicolumn{1}{|c|}{ Medidas correctoras } \\
\hline - Falta de consultores en el tema de Autoconcepto. & $\begin{array}{l}\text { - Puesta en marcha de un programa de ínterconsulta en temas } \\
\text { de autoconcepto. Las enfermeras de los E.A.P. de Parla } \\
\text { dispusieron de un servicio de consulta telefónica 2 horas a la } \\
\text { semana, con la enfermera de salud mental, en caso de duda en } \\
\text { diagnostico de autoconcepto. }\end{array}$ \\
\hline $\begin{array}{l}\text { - Falta de espacios para poner en común } \\
\text { experiencias y dudas sobre el tema del } \\
\text { autoconcepto. }\end{array}$ & $\begin{array}{l}\text { - Puesta en marcha de sesiones clínicas de enfermería sobre } \\
\text { temas de autoconcepto para cada uno de los cuatro E.A.P. de } \\
\text { Parla, junto con la enfermera de Salud Mental. Las sesiones } \\
\text { clínicas tuvieron periodicidad mensual y una duración de una } \\
\text { hora cada una. }\end{array}$ \\
\hline
\end{tabular}

Los efectos perseguidos con las medidas correctoras se exponen en el Programa de apoyo y coordinación entre enfermería de Salud Mental y enfermería de Atención Primaria, dentro del apartado "objetivos".

El contenido del programa es el siguiente:

\section{COORDINACIÓN ENTRE ENFERMERÍA} DE SALUD MENTAL Y ENFERMERÍA DE E.A.P. DE PARLA. ACTIVIDADES DE APOYO

\section{Objetivos.}

1.- Mejorar las capacidades de los profesionales de enfermería de los E.A.P. del distrito de Parla para la detención y tratamiento de problemas de autoconcepto en la población a la que prestan sus servicios.

2.- Apoyar a los profesionales enfermeros de los E.A.P. en el establecimiento y seguimiento de los planes de cuidados específicos en los problemas de autoconcepto.

3.- Favorecer el abordaje integral de la persona desde sus aspectos físicos y mentales.

\section{Actividades.}

1.- Interconsulta a petición de la enfermera/o de Atención Primaria, para evaluación del caso objeto de consulta y orientación de las actividades.
2.- Seguimiento conjunto del plan de cuidados.

3.- Sesiones de enfermería en el E.A.P. referidas a temas relacionados con los planes de cuidados enfermeros y a la discusión de casos. Las sesiones tendrán una periodicidad mínima mensual. Cada E.A.P. elaborará un calendario anual, en coordinación con la enfermera especialista en salud mental de Parla.

\section{Circuito de derivación.}

La solicitud de interconsulta se realizará telefónicamente, contactando con la enfermera especialista en salud mental de la localidad de Parla.

El teléfono de contacto: Ana García 91 6056903. Los lunes y los viernes de 14 a 15 horas.

No obstante, se pueden utilizar otros sistemas de comunicación como el Fax 916054512 o el correo interno: Centro de Salud Mental de Parla.

\section{Sistema de registro.}

Salud Mental registrará las interconsultas realizadas y las sesiones efectuadas.

Las sesiones se incluirán en el plan de formación de cada E.A.P. como sesiones de enfermería.

\section{Intervención.}

En el periodo comprendido entre octubre y mayo de 1999 se realizaron las siguientes actividades:

- Un total de 17 interconsultas.

- Un total de 17 sesiones de enfermería en los 
distintos E.A.P.s de Parla, cuyos temas principales fueron:

- Problemas de sobrepeso relacionados con la baja autoestima. Fue el tema más tratado en número de sesiones en todos los E.A.P.s.

- Baja autoestima crónica en sus diversas manifestaciones.

- Conductas adictivas relacionadas con la baja autoestima.

- Baja autoestima en menores.

Es necesario señalar que anteriormente no se realizaba interconsultas ni sesiones clínicas de enfermería en diagnóstico de autoconcepto.

\section{Indicadores:}

- Incremento en términos absolutos de los diagnósticos de enfermería en el área de autoconcepto en el periodo acumulado de octubre de 1998 a mayo de 1999 respecto al periodo febrero-septiembre de 1998 en el conjunto de los Centros de Salud de Parla.

Los datos de los diagnósticos de enfermería serán proporcionados por la Dirección de Enfermería del Área X Éstos provienen del Registro mensual que desde todos los centros de salud se remiten a la citada Dirección de Enfermería, y que recogen los diagnósticos de enfermería efectuados en cada una de las ocho áreas diagnósticas contempladas en éstos, correspondientes a los ocho requisitos de autocuidado universal y que son efectuados por las enfermeras de dichos centros.

\section{Universo y muestra:}

Universo. Número de diagnósticos realizados en el área de autoconcepto en los cuatro centros de salud ubicados en el municipio de Parla (Centro de Salud "San Blas", Centro de Salud "Pintores", Centro de Salud "Isabel II-1" y Centro de Salud "Isabel II-2").

Muestra. Se empleará el universo sin muestreo.

\section{Marco temporal:}

- $1^{\text {a }}$. evaluación: Se realizó el 1-9-98, los datos de referencia fueron los diagnósticos de enfermería acumulados entre febrero y septiembre de 98, periodo en el que los diagnósticos de enfermería en el área de autoconcepto se realizaron sin ningún tipo de actuación.

- Medidas correctoras: Se efectuó entre octubre del año 98 y junio del año 99. Dichas medidas se repetirán a partir de entonces entre octubre y junio de los sucesivos años.

- La reevaluación se efectuó el 7-6-99, sobre los datos del número absoluto acumulado de diagnósticos de enfermería en el área de autoconcepto, realizados entre octubre del año 98 y mayo del año 99 , periodo este en el que habrá comenzado a realizarse la intervención.

\section{DIAGNÓSTICOS DE ENFERMERÍA. EQUIPO DE PARLA}

\section{Evaluación:}

Se realizó una evaluación interna y cruzada con datos retrospectivos respecto a los diagnósticos referidos al periodo febrero-septiembre 98 y concurrente en el caso de los datos del periodo octubre 98-mayo 99.

En el periodo febrero-septiembre 98 se realizaron 70 diagnósticos de autoconcepto, mientras que en el periodo octubre 98-mayo 99 se realizaron un total de 63 .

\section{Incremento en cifras absolutas.}

$63-70=-7$ diagnósticos en el periodo octubre 98 - mayo 99, respecto al periodo febrero-septiembre 98 .

\section{Incremento en cifras relativas.}

$1^{\text {a }}$. Evaluación: 70 diagnósticos de autoconcepto de 1.147 diagnósticos totales, lo que supone un $6,1 \%$

$2^{\mathrm{a}}$. Evaluación: 63 diagnósticos de autoconcepto de 1.142 diagnósticos totales, lo que supone un $5,5 \%$

Se constatan las dificultades de implantación del diagnóstico de autoconcepto en la atención primaria, basadas en los siguientes causas:

- Presión asistencial, que tiene relación con dos variables, la presión de la demanda y la presión de otros profesionales sanitarios, lo que hace que se disponga de poco tiempo. 
- Producto de este primer factor se suele priorizar los diagnósticos más orgánicos por estar habituadas las enfermeras en su historia profesional más habituadas a manejarlos y ser susceptibles de manejarse con criterios más técnicos.

- Falta de herramientas conceptuales y metodológicas para abordar este diagnóstico que, por otra parte, es difícil de protocolizar.

Para modificar estas dificultades se han puesto en marcha distintos mecanismos metodológicos:

- Grupos de trabajo.

- Colaboración de manera más directa con las enfermeras de los Centros de Salud en interconsultas.

- Exposición de casos prácticos sobre el tema de autoconcepto.

- Reforzar y ampliar la consultoría de autoconcepto.

- Realización de cursos monográficos teóricoprácticos sobre él diagnóstico de autoconcepto.

Realizadas los anteriores procedimientos, ante las diversas resistencias a la implantación del diagnóstico de autoconcepto, se analiza de nuevo la población estudiada, y para no distorsionar el estudio, decidí aplicar el mismo sistema, esto es, la aplicación de un nuevo plan de mejora que estoy elaborando actualmente.

\section{BIBLIOGRAFÍA}

- Área 10. Atención Primaria del Insalud de Madrid. Protocolo asistencial. Catálogo de Diagnóstico de Enfermería y Manual de Planes de Cuidados. "Manual Interno". (2000) Madrid.

- Área 10. Atención Primaria del Insalud de Madrid. Memoria (1999) Gerencia del Área 10 del Insalud. Madrid.

- Cavanagh, S.J. (1.993) Modelo de Orem. Aplicación práctica. Masson-Salval, Madrid.

- Documentación del Curso "Gestión de la Calidad en los Servicios de Salud" (No publicado) (1998) Facultad de Medicina. Universidad de Murcia. Murcia.

- Lupiani Giménez, M.; Marín Toro, J.M.; Gala LEón, F.J. (1994) Presentación de un instrumento de recogida de datos y de un método de análisis, para valorar las necesidades de cuidado según $\mathrm{D}$. Orem. Enfermería Científica. $N^{\circ} 142-143$, pp. $75-$ 80.

- Orem, D. (1993) Modelo de Orem. Conceptos de Enfermería en la práctica. Masson-Salvat, Madrid.

\section{Diagnósticos de Enfermería. Área de Autoconcepto. Parla Actividad acumulada de febrero a septiembre 1998}

$\begin{array}{cc}\text { E.A.P. } & \text { Diagnósticos totales } \\ \text { S. Blas } & 266 \\ \text { Pintores } & 452 \\ \text { Isabel II }-1 & 105 \\ \text { Isabel II }-2 & 324 \\ \text { Total } & 1.147\end{array}$

Diagnósticos autoconcepto
4
33
8
25
70

$\%$ del total

1,5

7,3

7,6

7,7

6,1

Fuente: Dirección de Enfermeria. Area 10

$\begin{array}{cccc}\text { E.A.P. } & \text { Diagnósticos totales } & \text { Diagnósticos autoconcepto } & \text { \% Total } \\ \text { San Blas } & 329 & 11 & 3,3 \% \\ \text { Pintores } & 430 & 24 & 5,6 \% \\ \text { Isabel II }-1 & 119 & 4 & 3,3 \% \\ \text { Isabel II }-2 & 564 & 24 & 4,2 \% \\ \text { Total } & 1442 & 63 & 5,5 \%\end{array}$

